\title{
Schutzgruppen zur Synthese von Diaza-cyclophanen nach dem „Rigid-Group-Principle“
}

\author{
Ulrike Funke und Hans-Friedrich Grützmacher* \\ Fakultät für Chemie der Universität Bielefeld, \\ Postfach 8640, D-4800 Bielefeld
}

Eingegangen am 9. Februar 1989

Key Words: Diaza[n.2]cyclophan-enes / Rigid-group principle

\begin{abstract}
4,4-Dimethyl-1,2-bis(4-methylphenyl)-3,5-pyrazolidindion (4), 1,2Bis(4-methylphenyl)perhydro-3,6-pyridazindion (5) und 1,2-Bis(4methylphenyl)perhydro-3,7-diazepindion (6) wurden durch Kondensation von p-Hydrazotoluol mit den entsprechenden Dicarbonsäuredichloriden dargestellt und durch Photobromierung in die Brommethylderivate 7-9 übergeführt. Die Eignung von 7-9 als Vorstufen für die Synthese von 2-Thia-10,11-diaza[3.2]paracyclophan-10-en (2) und 2,5-Dithia-13,14-diaza[6.2]paracyclophan-13-en (3) wurde geprifft. Die Darstellung von 2 gelang unabhängig von der Ringgröße und Konformation von 7-9 nicht. 3 wird dagegen in allen Fällen glatt gebildet, wobei sich der Dimethylmalonylrest wegen der guten Zugänglichkeit, Stabilität und leichten Abspaltbarkeit im alkalischen Milieu besonders bewährt.
\end{abstract}

Die 2-Thia-10,11-diaza[3.2]cyclophane 1 und 2 sind als kurz verbrückte Heteracyclophane interessante Moleküle ${ }^{1\}}$. Als gemeinsame Untereinheit haben 1 und 2 eine Azobenzolgruppe, die aufgrund der Substitution in meta- oder paraStellung in cis-Konfiguration fixiert ist. In dieser Anordnung liegen die Benzolringe relativ dicht übereinander. Das Metaderivat 1 versucht der hieraus resultierenden Spannung aufgrund seiner konformativen Beweglichkeit durch Einstellung eines syn/anti-Gleichgewichts auszuweichen ${ }^{2)}$. Im Gegensatz hierzu ist das Paraderivat 2 ein ziemlich starres Molekül mit einer deutlich größeren Spannungsenergie. Mit Hilfe des MMPMI-Programms ${ }^{3)}$ wird für $2 \mathrm{im}$ Vergleich zu 1 eine Erhöhung der Spannungsenergie von $146 \mathrm{~kJ} / \mathrm{mol}$ (syn-1) auf $197 \mathrm{~kJ} / \mathrm{mol}$ berechnet. Während das 2-Thia10,11-diaza[3.2]metacyclophan-10-en (1) bereits auf zwei getrennten Synthesewegen dargestellt worden ist ${ }^{1,4}$, gelang bisher nur die Synthese des $N, N^{\prime}$-Phthaloylderivats des Paraisomeren 2 durch Ausnutzen eines "Caesiumeffekts" ${ }^{5)}$ bei der Cyclisierung nach Schema ${ }^{6}$.

Schema 1

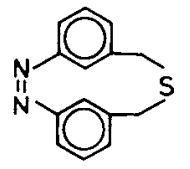

1

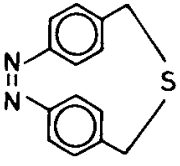

2

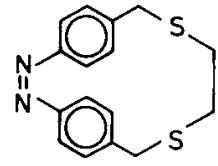

3
Protecting Groups for the Synthesis of Diaza-cyclophanes by the Rigid-Group Principle

4,4-Dimethyl-1,2-bis(4-methylphenyl)-3,5-pyrazolidinedione (4), 1,2-bis(4-methylphenyl)perhydro-3,6-pyridazinedione (5), and 1,2bis(4-methylphenyl)perhydro-3,7-diazepinedione (6) have been prepared by condensation of $p$-hydrazotoluene with the appropriate dicarbonyl dichlorides and then converted into the corresponding bis(bromomethyl) derivatives $7-9$ by photobromination. 7-9 have been tested as starting compounds for the synthesis of 2-thia-10,11-diaza[3.2]paracyclophan-10-ene (2) and 2,5-dithia13,14-diaza[6.2]paracyclophan-13-ene (3). In no case 2 has been obtained, independent of the ring size and conformation of 7-9. However, 3 was readily formed from all precursors; the dimethylmalonyl moiety proved to be convenient as a protecting group because of its ease of preparation, its stability, and its facile cleavage by alkali.<smiles>CC(=O)OCc1ccc(NNc2ccc(COC(C)=O)cc2)cc1</smiles><smiles>CC(=O)OCc1ccc(N2C(=O)c3ccccc3[C@@H]2O)cc1</smiles><smiles>[B]Cc1ccc(N2C(=O)c3ccccc3C2=O)cc1</smiles>
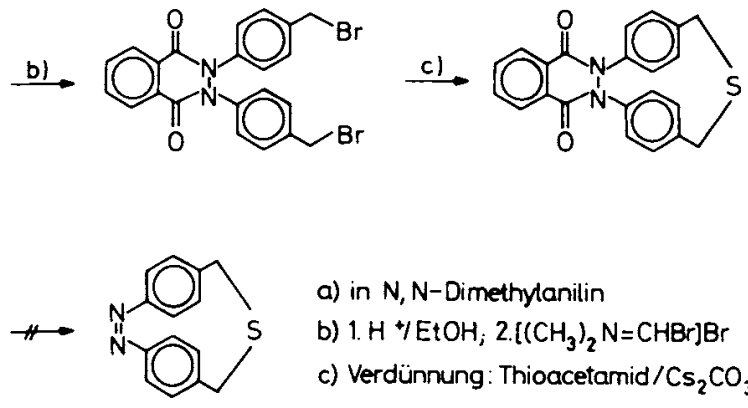

a) in $\mathrm{N}, \mathrm{N}-$ Dimethylanilin

b) $1 . \mathrm{H}+\mathrm{EtOH} ; 2 .\left(\left(\mathrm{CH}_{3}\right)_{2} \mathrm{~N}=\mathrm{CHBr}\right] \mathrm{Br}$

c) Verdünnung: Thioacetamid/ $\mathrm{Cs}_{2} \mathrm{CO}_{3} / \mathrm{DMF}$

2

Die Freisetzung des Diaza-cyclophans 2 durch Abspaltung des Phthaloylrestes gelang jedoch nicht. Im Fall von 1 konnte die Synthese durch Verwendung einer Dimethylmalonylschutzgruppe erheblich verkürzt und mit gesteigerter Ausbeute durchgeführt werden ${ }^{4}$. Bei Verlängerung der „Thiamethylen"-Brücke ist mit dieser Schutzgruppe auch die Synthese von Diaza-paracyclophanen möglich ${ }^{7)}$, obwohl z. B. das 2,5-Dithia-13,14-diaza[6.2]paracyclophan-13-en (3) $\mathrm{mit} 161 \mathrm{~kJ} / \mathrm{mol}$ bereits eine ähnliche Spannungsenergie besitzt wie 1. Es erschien daher möglich, durch Variation 
der Schutzgruppe auch $\mathbf{2}$ darzustellen. In dieser Arbeit wird über das Ergebnis dieser Versuche berichtet.

\section{Syntheseprinzip}

Zur Synthese von Thia- und Dithia-diaza-cyclophanen hat sich die kombinierte Anwendung des VerdünnungsprinSchema 2<smiles>Cc1ccc(NN(Cc2ccccc2)N2CPCN(Cc3ccccc3)C2)cc1</smiles>

$\Delta$
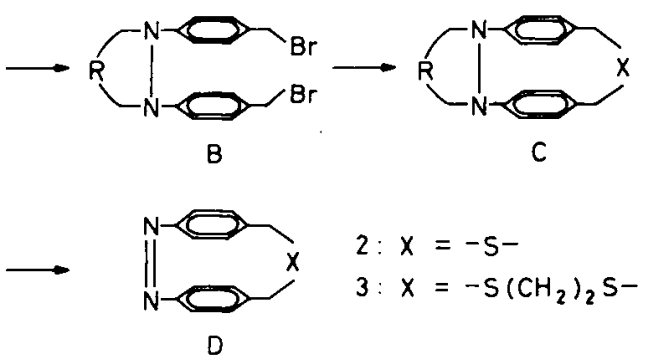

\begin{tabular}{|c|c|c|c|c|}
\hline & A & B & & \\
\hline$R^{x}$ & & & $-5-$ & $x^{\left(C_{2}\right)_{2} \mathrm{~S}-}$ \\
\hline & 4 & 7 & 10 & 13 \\
\hline & 5 & 8 & 11 & 14 \\
\hline & 6 & 9 & 12 & 15 \\
\hline
\end{tabular}

$z_{i p s^{8)}}$ und des Rigid-Group-Principle ${ }^{9)}$ als besonders geeignet erwiesen. Ausgehend von $p$-Hydrazotoluol wurde nach dem Syntheseprinzip in Schema 2 verfahren, bei dem die empfindliche Azo-(oder Hydrazo-)Brücke vorgegeben und durch eine Schutzgruppe eine sterische Fixierung der Aromaten in cis-Anordnung erzielt wird. Nach einer geeigneten Funktionalisierung der Tolylgruppen läßt sich dann der Ringschluß unter sterisch günstigen Bedingungen vollziehen. Die Wahl der Schutzgruppe ist für den Erfolg der Synthese der Thiaund Dithia-diaza-cyclophane von entscheidender Bedeutung. Verlangt wird Stabilität während der nachfolgenden Syntheseschritte, auch müssen die empfindlichen Diaza-cyclophane die Abspaltung der Schutzgruppe überstehen. Aliphatische Schutzgruppen können im Vergleich zur aromatischen Phthaloylgruppe im alkalischen Medium wesentlich leichter wieder entfernt werden ${ }^{4)}$ und sollten den Vorteil haben, daß durch die Ringgröße des Dicarbonsäurehydrazids der Abstand der Tolylgruppen im $N, N^{\prime}$-geschützten Hydrazotoluol variiert werden kann. Als Schutzgruppe wurden daher die Dimethylmalonyl-, die Succinyl- und die Glutarylgruppe ausgewählt und die $N, N^{\prime}$-disubstituierten Heterocyclen 4,5 und 6 auf ihre Eignung als Vorstufen für Diazaparacyclophane überprüft. Sie werden durch Kondensation der entsprechenden Dicarbonsäuredichloride mit $p$-Hydrazotoluol in $\mathrm{CHCl}_{3}$ erhalten, wobei 4-(Dimethylamino)pyridin als Katalysator eingesetzt wird.

Die Ausbeute der Kondensationsreaktion sinkt erwartungsgemä $ß$ mit zunehmender Ringgröße. Durch selektive Bromierung von 4-6 mit $N$-Bromsuccinimid sind die entsprechenden Dibromide 7, 8 und 9 in reiner Form erhältlich. 4-6 unterscheiden sich in den Bindungswinkeln am Stickstoff und damit in ihren Abständen der Tolylgruppen voneinander. Dreiding-Modelle zeigen, daß die Abstände zwischen den Tolylgruppen in der Reihe von $\mathbf{4}$ nach $\mathbf{6}$ deutlich abnehmen. Uberträgt man diese Verhältnisse auf die Dibro-

Tab. 1. Cyclisierungsversuche zu den $N, N^{\prime}$-geschützten 2-Thia-10,11-diaza[3.2] paracyclophanen 10-12

\begin{tabular}{|c|c|c|c|c|}
\hline $\begin{array}{l}\text { Cyclisierungs- } \\
\text { bedingungen }^{\text {a) }}\end{array}$ & Schwefelquelle & 7 & $\begin{array}{c}\text { Ergebnis bei } \\
\mathbf{8}\end{array}$ & 9 \\
\hline $\begin{array}{l}1 \mathrm{~K}-\mathrm{VP} \\
\mathrm{DMF}, 55^{\circ} \mathrm{C}\end{array}$ & $\mathrm{Na}_{2} \mathrm{~S} \cdot 9 \mathrm{H}_{2} \mathrm{O}$ & Polymere & - & Polymere \\
\hline $\begin{array}{l}2 \mathrm{~K}-\mathrm{VP}, \\
\mathrm{DMF} / \mathrm{Cs}_{2} \mathrm{CO}_{3}, 55^{\circ} \mathrm{C}\end{array}$ & Thioacetamid & Polymere & Polymere & Polymere \\
\hline $\begin{array}{l}3 \mathrm{~K}-\mathrm{VP} \text {, } \\
\text { Benzol/EtOH, } 20^{\circ} \mathrm{C}\end{array}$ & Thioacetamid & $\begin{array}{l}\text { Ethylether, } \\
\text { Monohydrazid, } \\
\text { Polymere }\end{array}$ & $\begin{array}{l}\text { Ethylether, } \\
\text { Monohydrazid, } \\
\text { Polymere }\end{array}$ & - \\
\hline $\begin{array}{l}\text { 1K-VP, } \\
\text { Benzol/EtOH, } 20^{\circ} \mathrm{C}\end{array}$ & $\mathrm{Na}_{2} \mathrm{~S} \cdot 9 \mathrm{H}_{2} \mathrm{O}$ & - & $\begin{array}{l}\text { Ethylether, } \\
\text { Monohydrazid, } \\
\text { Polymere }\end{array}$ & - \\
\hline $\begin{array}{l}3 \mathrm{~K}-\mathrm{VP} \\
\text { Benzol/EtOH, } 80^{\circ} \mathrm{C}\end{array}$ & Thioacetamid & $\begin{array}{l}\text { Ethylether, } \\
\text { Polymere }\end{array}$ & - & - \\
\hline $\begin{array}{l}\text { 2K-VP, } \\
\text { THF } / 18 \mathrm{C} 6,80^{\circ} \mathrm{C}\end{array}$ & Thioacetamid & - & Polymere & Polymere \\
\hline $\begin{array}{l}2 \mathrm{~K}-\mathrm{VP} \\
\text { Benzol } / 18 \mathrm{C} 6,80^{\circ} \mathrm{C}\end{array}$ & Thioacetamid & $\begin{array}{l}\text { nur geringe } \\
\text { Umsetzung }\end{array}$ & $\begin{array}{l}\text { nur geringe } \\
\text { Umsetzung }\end{array}$ & $\begin{array}{l}\text { nur geringe } \\
\text { Umsetzung }\end{array}$ \\
\hline $\begin{array}{l}1 \mathrm{~K}-\mathrm{VP} \\
\text { Benzol } / 18 \mathrm{C} 6,80^{\circ} \mathrm{C}\end{array}$ & $\mathrm{Na}_{2} \mathrm{~S} \cdot 9 \mathrm{H}_{2} \mathrm{O}$ & - & $\begin{array}{l}\text { nur geringe } \\
\text { Umsetzung }\end{array}$ & - \\
\hline
\end{tabular}

a) $n \mathrm{~K}-\mathrm{VP}=n$-Komponenten-Verdünnungs-Prinzip $(n=1-3), 18 \mathrm{C} 6=18$-Krone- 6 . 
mide, so sollte sich 9 am besten für die Cyclisierung zu Thiadiaza-paracyclophanen eignen.

\section{Cyclisierungsversuche zu den $N, N^{\prime}$-geschützten 2-Thia- 10,11-diaza[3.2]paracyclophanen 10-12}

In Tab. 1 sind die Ergebnisse der Cyclisierungsversuche mit 7-9 durch Einführung eines S-Atoms bei verschiedenen Reaktionsbedingungen zusammengefa $ß t$. Eine Cyclisierung zum gewünschten Derivat des Thia-diaza[3.2]paracyclophans $\mathbf{2}$ ist auch bei Verwendung aliphatischer Schutzgruppen weder mit $\mathrm{Na}_{2} \mathrm{~S}$ noch mit Thioacetamid als Schwefelquelle gelungen. Die Dibromide 7-9 reagieren bereits bei Raumtemperatur mit Thioacetamid unter Bildung der Bisthiuroniumsalze. Die Reaktionen mußten daher entweder nach dem Einkomponenten-Verdünnungsprinzip (1K-VP), bei dem das Dibromid langsam in eine Lösung von $\mathrm{Na}_{2} \mathrm{~S}$ oder Thioacetamid und $\mathrm{KOH}$ getropft wurde, oder nach dem Zweikomponenten-Verdünnungsprinzip (2K-VP) durchgeführt werden, bei dem Dibromid und Thioacetamid durch zwei getrennte Tropftrichter zugegeben wurden. Schließlich wurden auch Cyclisierungsversuche nach dem Dreikomponenten-Verdünnungsprinzip (3K-VP) durchgeführt, bei dem auch die Base durch einen zusätzlichen (dritten) Tropftrichter zugegeben wurde. Trotzdem entstanden stets nur polymere Substanzen. Benzol/Ethanol-Gemische als Lösungsmittel ergaben dazu regelmäßig als Hauptprodukt die von 7-9 durch Austausch der Bromatome abgeleiteten Diethylether neben Säurehydraziden durch partielle Hydrolyse der cyclischen Bishydrazidgruppierung. Reaktionen in Dimethylformamid und Kaliumcarbonat als Base brachten keine Verbesserung. Als Ursache für die ausbleibende Cyclisierung wird bei 7 ein zu großer Abstand der Brommethylsubstituenten angenommen, denn im Gegensatz zum Phthaloylderivat sind die 4-(Brommethyl)phenyl-Substituenten am heterocyclischen Fünfring von 7 auseinander gespreizt. Dies gilt für 8 und 9 jedoch nicht, so daß die Cyclisierung dieser Dibromide möglicherweise durch die Instabilität des heterocyclischen Ringes gegenüber nucleophilen Reagenzien insbesondere in Dimethylformamid als Lösungsmittel verhindert wird. Dies läßt sich durch Cyclisierungsversuche mit 1,2-Ethandithiol überprüfen.

\section{Cyclisierungsversuche zu $\quad N, N^{\prime}$-disubstituierten 2,5-Dithia-} 13,14-diaza[6.2] paracyclophanen $13-15$

Die Umsetzung der Dibromide 7-9 mit 1,2-Ethandithiol führt glatt zur Cyclisierung unter Bildung der $N, N^{\prime}$-disubstituierten Dithia-diaza[6.2]paracyclophane 13, 14 und 15. Dies ermöglicht einen Vergleich der Schutzgruppen verschiedener Ringgröße bei der Cyclophansynthese. Die besten Ausbeuten bei diesen Cyclisierungen werden überraschenderweise im unpolaren Lösungsmittel Benzol mit Kaliumcarbonat als Base und 18-Krone-6 als Phasentransferkatalysator erhalten. Bei Verwendung von Dimethylformamid und Caesiumcarbonat überwiegen dagegen unerwünschte Nebenreaktionen. Dies bestätigt die vermutete Instabilität von 7-9 in polaren Lösungsmitteln. Die Ausbeute bei der Cyclisierungsreaktion zum Perhydrodiazepindion-
Derivat 15 ist entgegen der Erwartung deutlich geringer als bei 13 und 14. Vermutlich ist der Abstand zwischen den Brommethylgruppen bei der Vorstufe 9 für den Einbau des 1,2-Ethandithiols zu klein. Dies wird auch bei einem Vergleich der ${ }^{1}$ H-NMR-Spektren der Cyclophane 13-15 deutlich.

Auffallend ist das komplexe Aufspaltungsmuster im Bereich der aliphatischen sowie der aromatischen Protonen im NMR-Spektrum von 15. Während in den Spektren von 13 und 14 die benzylischen Protonen sowie die Ethylenprotonen der Disulfidbrücke als scharfe Singuletts auftreten, ist im Spektrum von 15 ein AB-System für die benzylischen Protonen und ein $\mathrm{AA}^{\prime} \mathrm{BB}^{\prime}$-System für die Ethylenprotonen der Disulfidbrücke zu erkennen. Außerdem spalten die Methylenprotonen im Perhydrodiazepindion-Ring von $15 \mathrm{zu}$ einem $\mathrm{AA}^{\prime} \mathrm{BB}^{\prime} \mathrm{CC}^{\prime}$-System mit einer geminalen Kopplungskonstanten von $J_{\mathrm{AB}}=-12.8 \mathrm{~Hz}$ auf. Auch im aromatischen Bereich treten deutliche Unterschiede auf. Im Spektrum von 13 erscheinen die aromatischen Protonen als Multiplett eines $\mathrm{AA}^{\prime} \mathrm{BB}^{\prime}$-Systems mit einem Zentrum bei $\delta=$ 7.10. Im Fall von 14 sind diese Signale aufgrund zufälliger Isochronie zu einem Singulett verschmolzen. Demgegenüber sind die Ringprotonen bei 15 in drei Signalgruppen bei $\delta=$ $7.34,7.04$ und 6.84 mit einem Intensitätsverhältnis von $1: 2: 1$ in Form eines ABCD-Systems aufgespalten. Die Aufspaltungsmuster der aromatischen und aliphatischen Protonen von 15 deuten auf eine stark eingeschränkte Beweglichkeit dieses geschützten Dithia-diaza-cyclophans sowohl im $\mathrm{Cy}$ clophanring als auch im Heterocyclus hin.

\section{Abspaltung der Schutzgruppen}

Im Gegensatz zu der Phthalazindiongruppe lassen sich die gesättigten Dicarbonsäurehydrazidgruppen in 13-15 unter alkalischen Bedingungen leicht spalten. Wie bereits beschrieben ${ }^{7}$, werden die besten Ausbeuten bei Verwendung von Natriumethanolat in Dimethylformamid erzielt. Die Reaktion muß unter Stickstoff durchgeführt werden, um die Bildung von Azoxyverbindungen zu vermeiden. Unter diesen Bedingungen wird zunächst das gesättigte 2,5-Dithia13,14-diaza[6.2]paracyclophan (16) gebildet, das in Lösung entweder mit Luftsauerstoff oder durch $\mathrm{MnO}_{2}$ zu 3 oxidiert werden kann (Schema 3).

\section{Schema 3}

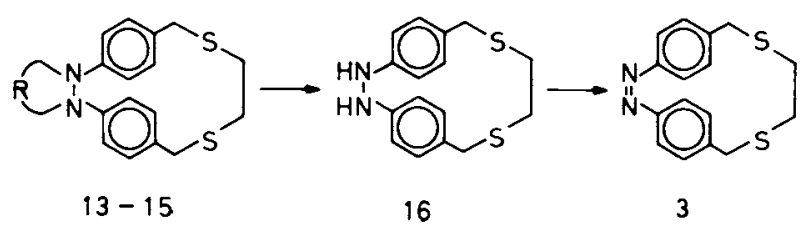

Diese Versuche zeigen, da $\beta$ aliphatische Schutzgruppen zur Darstellung makrocyclischer Dithia-diaza-paracyclophane von Vorteil sind. So lassen sich die Dimethylmalonyl-, die Succinyl- und die Glutarylschutzgruppe im alkalischen Medium wesentlich leichter wieder entfernen als die bisher verwendete Phthaloylschutzgruppe. Von den untersuchten 
Schutzgruppen ist die Dimethylmalonylgruppe aufgrund der guten Zugänglichkeit, der Stabilität und der konformativen Eigenschaften am besten zur Darstellung größerer Dithiadiaza-cyclophane geeignet. Unabhängig von der Ringgröße oder der Konformation ermöglicht jedoch keine der aliphatischen Schutzgruppen die Darstellung des Thia-diaza-paracyclophans 2. Vermutlich ist die Cyclisierung unter Einbau eines Schwefelatoms nur unter solchen experimentellen Bedingungen möglich, bei denen die Cyclophanvorstufen mit gesättigten Heterocyclen, insbesondere die Perhydrodiazepindion-Derivate, nicht stabil sind.

Wir danken Herrn K. P. Mester für die Hochfeld-NMR-Spektren und Herrn E. Gärtner für die Massenfeinbestimmungen. Dem Fonds der Chemischen Industrie danken wir für finanzielle Unterstützung.

\section{Experimenteller Teil}

Schmelzpunkte (unkorrigiert): Büchi 512. - IR-Spektren: Infrarotgitterspektrometer Modell Perkin-Elmer 377. - UV-Spektren: Spektrometer Modell Beckman 25. - Massenspektren: MAT 311 A, Elektronenstoß-Ionisation bei $70 \mathrm{eV}$. Die Massenfeinbestimmungen zur Elementaranalyse wurden mit demselben Gerät mit einer Auflösung $\Delta m / m=6000$ durchgeführt. - Elementaranalysen: Elementaranalytisches Zentrallabor der Fakultät für Chemie, Universität Bielefeld. - ${ }^{1} \mathrm{H}-\mathrm{NMR}$-Spektren und ${ }^{13} \mathrm{C}-\mathrm{NMR}-\mathrm{Spek}-$ tren: Bruker-AM-300-Spektrometer und Bruker-WP-80-Spektrometer (Lösungsmitel $\mathrm{CDCl}_{3}$, Deuterierungsgrad 98.9\%; mit TMS als internem Standard). - Dünnschichtchromatographie: MerckDC-Aluminiumfolien Kieselgel $60 \mathrm{~F}_{254}$, Schicht $0.2 \mathrm{~mm}$. - Säulenchromatographie: Merck-Kieselgel 60, Korngröße unter 0.063 mm. - MPLC-Trennungen: Pumpe Duramat 80 (Fa. Cfg) und Merck-Fertigsäule Si $60(40-63 \mu \mathrm{m}) .-n \mathrm{~K}-\mathrm{VP}=n$-Komponenten-Verdünnungs-Prinzip $(n=1-3)$.

\section{Kondensationsreaktionen}

Die nachstehenden Reaktionen wurden in sorgfältig getrockneten Lösungsmitteln durchgeführt. Die verwendeten Apparaturen wurden vor Gebrauch gründlich mit Stickstoff gespült, und während der Kondensationsreaktionen wurde ein schwacher Stickstoffstrom durch die gesamte Apparatur geleitet.

4,4-Dimethyl-1,2-bis(4-methylphenyl)-3,5-pyrazolidindion (4): $21 \mathrm{ml}(0.1 \mathrm{~mol})$ Dimethylmalonsäuredichlorid wurden unter Feuchtigkeitsausschlu $B$ bei $-15^{\circ} \mathrm{C}$ in eine Mischung aus $50 \mathrm{ml}$ Pyridin und $100 \mathrm{ml} \mathrm{CHCl}$ getropft, und etwas 4-(Dimethylamino)pyridin wurde als Katalysator zugegeben. Dann wurden $21 \mathrm{~g}(0.1 \mathrm{~mol})$ p-Hydrazotoluol in $250 \mathrm{ml} \mathrm{CHCl}_{3}$ bei $10 \mathrm{C}$ langsam zugetropft. Nach ca. 12stdg. Rühren und Erwärmen auf Raumtemp. wurde mit eiskalter verdünnter $\mathrm{HCl}(500 \mathrm{ml})$ ausgeschüttelt, mit 5 proz. $\mathrm{NaHCO}_{3}$-Lösung neutralisiert (Farbumschlag von Tiefrot nach Hellgelb), mit Wasser gewaschen und mit $\mathrm{Na}_{2} \mathrm{SO}_{4}$ getrocknet. Nach Entfernen des Lösungsmittels i. Vak. wurde der gelbe Rückstand zweimal aus Diethylether umkristallisiert; Ausb. $17.2 \mathrm{~g}(56 \%)$ farblose Nadeln, Schmp. $148^{\circ} \mathrm{C} ; R_{\mathrm{f}}=0.4$ (Petrolether/Essigester, 4:1), $0.3\left(\mathrm{CH}_{2} \mathrm{Cl}_{2}\right) .-\mathrm{IR}(\mathrm{KBr}): \mathrm{v}=1720 \mathrm{~cm}^{-1}(\mathrm{C}=\mathrm{O}), 1310(\mathrm{C}-\mathrm{N})$. ${ }^{1} \mathrm{H}$-NMR $(80 \mathrm{MHz}): \delta=7.14(\mathrm{~m}, 8 \mathrm{H}$, aromat. $\mathrm{H}), 2.27(\mathrm{~s}, 6 \mathrm{H}$, aromat. $\left.\mathrm{CH}_{3}\right), 1.48\left(\mathrm{~s}, 6 \mathrm{H}, \mathrm{CH}_{3}\right) .-\mathrm{MS}: m / z(\%)=308(100)$ $\left[\mathrm{M}^{+\cdot}\right], 210(13), 147(34), 132(88), 119(39), 104(16), 105(16), 91$ (15).

$$
\begin{array}{ll}
\mathrm{C}_{19} \mathrm{H}_{20} \mathrm{~N}_{2} \mathrm{O}_{2} \text { (308.4) } & \text { Ber. C } 74.0 \text { H } 6.5 \text { N } 9.1 \\
& \text { Gef. C } 73.8 \text { H } 6.5 \text { N } 9.2
\end{array}
$$

1,2-Bis (4-methylphenyl) perhydro-3,5-pyridazindion (5): Eine Lösung von $10.5 \mathrm{~g}$ (0.05 mol) $p$-Hydrazotoluol in $150 \mathrm{ml}$ Toluol und eine Lösung von $11 \mathrm{ml}$ (0.1 mol) Bernsteinsäuredichlorid ( $d=1.41$ $\mathrm{g} / \mathrm{ml}$ ) in $150 \mathrm{ml}$ Toluol wurden über zwei Feindosiertrichter synchron in eine kräftig gerührte Lösung aus 11 Toluol, $10 \mathrm{ml}$ Pyridin und einer Spatelspitze 4-(Dimethylamino)pyridin getropft. Während der Reaktionszeit (DC-Kontrolle) von etwa $6 \mathrm{~h}$ färbte sich die Lösung tiefblau. Nach Einengen des Reaktionsansatzes bis auf ca. $150 \mathrm{ml}$ wurde über feines Kieselgel (Korngröße unter $0.063 \mathrm{~mm}$ ) filtriert und das Kieselgel einige Male bis zum Verschwinden der gelben Farbe mit Toluol gewaschen. Die dunkelblaue Kieselgelschicht am Start wurde verworfen und das Produkt mit kleinen Portionen eines Toluol/Aceton-Gemisches (4:1) von der Säule gespült. Die Fraktionen wurden eingeengt, und der Rückstand wurde aus Ethanol umkristallisiert; Ausb. $7.8 \mathrm{~g} \mathrm{(53 \% )} \mathrm{farblose} \mathrm{Nadeln,}$ Schmp. $211-213^{\circ} \mathrm{C}$ (Lit. ${ }^{10)} 209-210^{\circ} \mathrm{C}$ ), $R_{\mathrm{f}}=0.4$ (Toluol/Aceton, 4:1). - IR (KBr): v $=1680 \mathrm{~cm}^{-1}(\mathrm{C}=\mathrm{O}), 1350(\mathrm{C}-\mathrm{N}) .-{ }^{1} \mathrm{H}-$ NMR (80 MHz): $\delta=7.13\left(\mathrm{~m}, 8 \mathrm{H}\right.$, aromat. H), $2.90\left(\mathrm{~s}, 4 \mathrm{H}, \mathrm{COCH}_{2}\right)$, $2.24\left(\mathrm{~s}, 6 \mathrm{H}, \mathrm{CH}_{3}\right) .-\mathrm{MS}: m / z(\%)=294(100)\left[\mathrm{M}^{+\cdot}\right], 211(36)$, 189 (8), 188 (9), 160 (13), 133 (14), 132 (9), 119 (8), 107 (17), 106 (19), 105 (24), 91 (51).

\section{$\mathrm{C}_{18} \mathrm{H}_{18} \mathrm{~N}_{2} \mathrm{O}_{2} \quad$ Ber. 294.1368 Gef. 294.1368 (MS)}

1,2-Bis(4-methylphenyl)perhydro-3,7-diazepindion (6): Eine Lösung von $5.25 \mathrm{~g}(0.025 \mathrm{~mol}) \mathrm{p}$-Hydrazotoluol in $150 \mathrm{ml}$ Toluol und eine Lösung von $6.4 \mathrm{ml}(0.05 \mathrm{~mol})$ Glutarsäuredichlorid $(d=1.32$ $\mathrm{g} / \mathrm{ml})$ in $150 \mathrm{ml}$ Toluol wurden über zwei Feindosiertrichter synchron in eine Lösung aus 21 Toluol, $10 \mathrm{ml}$ Pyridin und einer Spatelspitze 4-(Dimethylamino)pyridin getropft. Nach etwa $6 \mathrm{~h}$ wurde die Zugabe der Reaktanden mit den gleichen Mengen wiederholt. Die Reaktionsdauer betrug etwa $12 \mathrm{~h}$. Die gelbe Reaktionslösung wurde nach Ende der Reaktion (DC-Kontrolle) bis auf etwa $20 \mathrm{ml}$ eingeengt. Anschließende Flash-Chromatographie mit je $10 \mathrm{ml}$ Toluol zur Entfernung des Azotoluols sowie des Dikondensationsproduktes und Umkristallisation (Ethanol) lieferte reines 6; Ausb. $4.9 \mathrm{~g}$ $(32 \%)$ farblose Nadeln, Schmp. $143^{\circ} \mathrm{C}, R_{\mathrm{f}}=0.5$ (Toluol/Aceton, 4:1). - IR (KBr): $v=1680 / 1690 \mathrm{~cm}^{-1}(\mathrm{C}=\mathrm{O}), 1340(\mathrm{C}-\mathrm{N})$. ${ }^{1} \mathrm{H}$-NMR (300 MHz): $\delta=7.26 / 7.08$ (AA'BB'-System, $8 \mathrm{H}$, aromat. H), 3.0/2.61/2.38 [AA'BB'CC'-System, 6H, $\mathrm{CH}_{\mathrm{ax}} \mathrm{CO}(\mathrm{A}), \mathrm{CH}_{2}(\mathrm{C})$, $\left.\mathrm{CH}_{e q} \mathrm{CO}(\mathrm{B}),{ }^{1} J_{\mathrm{AB}}=-11.8 \mathrm{~Hz}\right], 2.24\left(\mathrm{~s}, 6 \mathrm{H}, \mathrm{CH}_{3}\right) .-\mathrm{MS}: m / z$ $(\%)=308(75]\left[\mathrm{M}^{+*}\right], 212(30), 211(84), 202(8), 175(15), 174(47)$, 133 (9), 132 (11), 119 (6), 107 (26), 91 (100).

$$
\mathrm{C}_{19} \mathrm{H}_{20} \mathrm{~N}_{2} \mathrm{O}_{2} \text { Ber. } 308.1525 \text { Gef. } 308.1524 \text { (MS) }
$$

\section{Bromierungen}

1,2-Bis [4-(brommethyl) phenyl J-4,4-dimethyl-3,5-pyrazolidindion (7): $\mathrm{Zu}$ einer Lösung von $9.2 \mathrm{~g}(0.03 \mathrm{~mol}) 4$ in $500 \mathrm{ml}$ trockenem $\mathrm{CH}_{2} \mathrm{Cl}_{2}$ wurden $10.7 \mathrm{~g}(0.06 \mathrm{~mol}) \mathrm{N}$-Bromsuccinimid sowie etwas Brom als Radikalstarter gegeben. Das Reaktionsgemisch wurde auf $-20^{\circ} \mathrm{C}$ gekühlt (Kryostat) und unter kräftigem Rühren $20 \mathrm{~h}$ mit einer 500-W-Photolampe bestrahlt. Der Reaktionsverlauf wurde dünnschichtchromatographisch sowie 'H-NMR-spektroskopisch kontrolliert. Nach Entfernen des Lösungsmittels im Rotationsverdampfer wurde der feste Rückstand mehrmals mit je $50 \mathrm{ml} \mathrm{CCl}{ }_{4}$ ausgelaugt und vom Unlöslichen abgenutscht. Aus der eingeengten $\mathrm{CCl}_{4}$-Lösung kristallisierten im Kühlschrank $7.5 \mathrm{~g}$ noch verunreinigtes Dibromid aus. Erneute Kristallisation lieferte reines Produkt; Ausb. $6.6 \mathrm{~g} \mathrm{(47 \% )} \mathrm{farblose,} \mathrm{körnige} \mathrm{Kristalle,} \mathrm{Schmp.} 171^{\circ} \mathrm{C} ; R_{\mathrm{f}}=$ $0.3\left(\mathrm{CH}_{2} \mathrm{Cl}_{2}\right), 0.8$ (Toluol/Aceton, 4:1). - IR (KBr): $v=1720 \mathrm{~cm}^{-1}$ $(\mathrm{C}=\mathrm{O}), 1300(\mathrm{C}-\mathrm{N}), 590(\mathrm{C}-\mathrm{Br}) .-{ }^{1} \mathrm{H}-\mathrm{NMR}(300 \mathrm{MHz}): \delta=$ 7.33 (s, $8 \mathrm{H}$, aromat. $\mathrm{H}), 4.42\left(\mathrm{~s}, 4 \mathrm{H}, \mathrm{CH}_{2}\right), 1.49\left(\mathrm{~s}, 6 \mathrm{H}, \mathrm{CH}_{3}\right)$. MS: $m / z(\%)=464 / 466 / 468(14 / 28 / 14)\left[\mathrm{M}^{+\cdot}\right], 385 / 387(98 / 100)$, 307 (20), 153 (20), 147 (10), 146 (88), 132 (87), 90 (36), 89 (20).

$$
\begin{array}{ll}
\mathrm{C}_{19} \mathrm{H}_{18} \mathrm{Br}_{2} \mathrm{~N}_{2} \mathrm{O}_{2}(466.2) & \text { Ber. C } 48.9 \text { H } 3.86 \text { N } 6.01 \\
& \text { Gef. C 48.3 H } 3.98 \text { N } 6.09
\end{array}
$$


1,2-Bis [4-(brommethyl) phenyl]perhydro-3,6-pyridazindion (8): Dargestellt wie 7 , jedoch bei $-40^{\circ} \mathrm{C}$, aus $5.00 \mathrm{~g}(0.017 \mathrm{~mol}) 5 \mathrm{in}$ $800 \mathrm{ml} \mathrm{CH} \mathrm{Cl}_{2}$ und $6.05 \mathrm{~g}(0.034 \mathrm{~mol}) \mathrm{N}$-Bromsuccinimid. Nach Ende der Reaktion wurde das $\mathrm{CH}_{2} \mathrm{Cl}_{2}$ vorsichtig im Rotationsverdampfer bis zur beginnenden Kristallisation entfernt und die Lösung mehrere Male mit Wasser extrahiert, um das in $\mathrm{CH}_{2} \mathrm{Cl}_{2}$ gelöste Succinimid abzutrennen. Die organische Phase wurde eingeengt und aus Toluol/Aceton umkristallisiert; Ausb. $2.4 \mathrm{~g} \mathrm{(30 \% )} \mathrm{farblose,}$ körnige Kristalle, Schmp. $205^{\circ} \mathrm{C}, \quad R_{f}=0.44$ (Toluol/Aceton, 4:1). - IR (KBr): $v=1700 \mathrm{~cm}^{-1}(\mathrm{C}=\mathrm{O}), 1290 / 1320(\mathrm{C}-\mathrm{N}), 595$ $(\mathrm{C}-\mathrm{Br}) .-{ }^{\prime} \mathrm{H}-\mathrm{NMR}(300 \mathrm{MHz}): \delta=7.34\left(\mathrm{AA}^{\prime} \mathrm{BB}^{\prime}-\right.$ System, $8 \mathrm{H}$, aromat. $\mathrm{H}), 4.38\left(\mathrm{~s}, 4 \mathrm{H}\right.$, benzyl. $\left.\mathrm{CH}_{2}\right), 2.92\left(\mathrm{~s}, 4 \mathrm{H}, \mathrm{CH}_{2} \mathrm{CO}\right)$ - MS: $m / z(\%)=450 / 452 / 454(5 / 18 / 7)\left[\mathrm{M}^{+\cdot}\right], 371 / 373(59 / 68), 292(11)$, 266/268 (12/12), $209(20), 188(56), 132$ (60), $106(100), 104(15), 90$ (22).

\section{$\mathrm{C}_{18} \mathrm{H}_{16} \mathrm{Br}_{2} \mathrm{~N}_{2} \mathrm{O}_{2} \quad$ Ber. 451.9559 Gef. 451.9559 (MS)}

1,2-Bis [4-(brommethyl) phenyl]perhydro-3,7-diazepindion (9): Dargestellt wie 8 aus $3.1 \mathrm{~g}(0.01 \mathrm{~mol}) 6$ in $400 \mathrm{ml} \mathrm{CH}_{2} \mathrm{Cl}_{2}$ und $3.6 \mathrm{~g}$ (0.02 mol) $N$-Bromsuccinimid. Anstelle der Kristallisation wurde eine Flash-Chromatographie mit Toluol/Aceton (4:1) durchgeführt. Nach Entfernen des Lösungsmittels wurde ein farbloser Feststoff erhalten, der aus Toluol/Aceton umkristallisiert wurde; Ausb. $1.2 \mathrm{~g}$ (25\%) farblose, körnige Kristalle, Schmp. $198^{\circ} \mathrm{C}$ (Zers.), $R_{f}=0.55$ (Toluol/Aceton, 4:1). - IR (KBr): $v=1690 \mathrm{~cm}^{-1}(\mathrm{C}=\mathrm{O}), 1350$ $(\mathrm{C}-\mathrm{N}), 600(\mathrm{C}-\mathrm{Br}) .-{ }^{1} \mathrm{H}-\mathrm{NMR}(300 \mathrm{MHz}): \delta=7.42 / 7.33$ (AA'BB'-System, 8H, aromat. $\mathrm{H}$ ), 4.39 (s, 4H, benzyl. $\mathrm{CH}_{2}$ ), 2.92/ 2.62/2.38 [ $\mathrm{AA}^{\prime} \mathrm{BB}^{\prime} \mathrm{CC}^{\prime}-$ System, $6 \mathrm{H}, \mathrm{CH}_{\mathrm{ax}} \mathrm{CO}(\mathrm{A}), \mathrm{CH}_{2}(\mathrm{C}), \mathrm{CH}_{\mathrm{eq}} \mathrm{CO}$ (B), $\left.{ }^{1} J_{2 \mathrm{AB}}=-12.1 \mathrm{~Hz}\right]$. - MS: $m / z(\%)=464 / 466 / 468(8 / 13 / 8)$ $\left[\mathrm{M}^{+\cdot}\right], 385 / 387(61 / 60), 252 / 254(9 / 8), 209(41), 208(10), 202(22)$, 184/186 (5/5), 174 (11), $146(11), 132$ (44), 107 (18), 106 (100), 105 (8), $104(11)$.

$$
\mathrm{C}_{19} \mathrm{H}_{18} \mathrm{Br}_{2} \mathrm{~N}_{2} \mathrm{O}_{2} \quad \text { Ber. } 465.9716 \text { Gef. } 465.9714 \text { (MS) }
$$

\section{Cyclisierungen}

Cyclisierungsversuche zu den $N, N^{\prime}$-geschützten 2-Thia-10,11-diaza[3.2] paracyclophanen 10-12. - a) In DMF nach dem $2 K-V P$ :

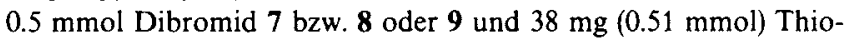
acetamid wurden in je $150 \mathrm{ml}$ getrocknetem und frisch destilliertem DMF gelöst und innerhalb von $10 \mathrm{~h}$ aus zwei Feindosiertropftrichtern unter Stickstoff und kräftigem Rühren simultan in eine Suspension aus $328 \mathrm{mg}$ ( $1 \mathrm{mmol})$ Caesiumcarbonat in $11 \mathrm{DMF}$ bei $55^{\circ} \mathrm{C}$ getropft. Das schwach gelbe Reaktionsgemisch wurde nach Ende der Zugabe noch $1 \mathrm{~h}$ bei $55^{\circ} \mathrm{C}$ gerührt und dann das Lösungsmittel i. Vak. bei dieser Temperatur entfernt. Der ölige, braune Rückstand wurde in Wasser aufgenommen und mehrere Male mit $\mathrm{CH}_{2} \mathrm{Cl}_{2}$ extrahiert. Die vereinigten organischen Phasen wurden einmal mit 5proz. $\mathrm{NH}_{4} \mathrm{Cl}$-Lösung und mehrfach mit ges. $\mathrm{NaCl}$-Lösung gewaschen und mit Natriumsulfat getrocknet. Nach Einengen des Lösungsmittels wurde eine Flash-Chromatographie mit Toluol/Aceton als Laufmittel durchgeführt. Die einzelnen Fraktionen wurden ${ }^{1} \mathrm{H}$-NMR-spektroskopisch und massenspektrometrisch untersucht; Ergebnisse s. Tab. 1.

b) In DMF nach dem $1 K$-VP: $0.5 \mathrm{mmol}$ Dibromid 7 bzw. 9 wurden in $150 \mathrm{ml}$ getrocknetem DMF gelöst und innerhalb von $10 \mathrm{~h} \mathrm{mit}$ Hilfe eines Feindosiertropftrichters unter kräftigem Rühren und Stickstoff in eine Suspension aus $120 \mathrm{mg}(0.5 \mathrm{mmol}) \mathrm{Na}_{2} \mathrm{~S} \cdot 9 \mathrm{H}_{2} \mathrm{O}$, $328 \mathrm{mg}(1 \mathrm{mmol})$ Caesiumcarbonat und 1 I DMF (frisch destilliert) bei $55^{\circ} \mathrm{C}$ getropft. Die Reaktion wurde auch ohne Caesiumcarbonat durchgeführt. Die Aufarbeitung erfolgte wie unter a); Ergebnisse s. Tab. 1.

c) In Benzol/Ethanol (2:1) nach dem 3K-VP: In einer Dreikomponenten-Verdünnungsprinzip-Apparatur nach Vögtle ${ }^{8 b)}$ wurde eine Lösung von $0.5 \mathrm{mmol}$ Dibromid $7 \mathrm{bzw}$. 8, eine Lösung von $38 \mathrm{mg}$
(0.51 mmol) Thioacetamid in je $150 \mathrm{ml}$ getrocknetem Benzol und eine Lösung von $60-120 \mathrm{mg}(1-2 \mathrm{mmol}) \mathrm{KOH}$ in $150 \mathrm{ml}$ absol. Ethanol innerhalb von ca. $10 \mathrm{~h}$ in ein heftig siedendes Lösungsmittelgemisch aus Benzol/Ethanol (2:1) getropft. Nach Ende der Zugabe wurde noch eine weitere Stunde gerührt. Nach Entfernen des Lösungsmittels i. Wasserstrahlvak. wurde der Rückstand mit $\mathrm{CHCl}_{3}$ extrahiert. Die $\mathrm{CHCl}_{3}$-Phase wird wie unter a) beschrieben aufgearbeitet; Ergebnisse s. Tab. 1

d) In THF oder Benzol nach dem 2K-VP: In einer Zweikomponenten-Verdünnungsprinzip-Apparatur wurden eine Lösung von $0.5 \mathrm{mmol}$ Dibromid 7 bzw. 8 oder 9 und eine Lösung von $38 \mathrm{mg}$ $(0.5 \mathrm{mmol})$ Thioacetamid in je $150 \mathrm{ml}$ getrocknetem THF oder Benzol innerhalb von ca. $10 \mathrm{~h}$ in eine kräftig gerührte und heftig siedende Suspension aus $1.4 \mathrm{~g}$ (10 mmol) Kaliumcarbonat und $200 \mathrm{mg}$ 18-Krone-6 in 11 THF oder Benzol getropft. Die Aufarbeitung erfolgte wie bei a); Ergebnisse s. Tab. 1.

13,14-Dimethylmalonyl)-2,5-dithia-13,14-diaza(6.2Jparacyclophan (13). - IK-VP mit DMF: Eine Lösung von $466 \mathrm{mg}$ (1 mmol)

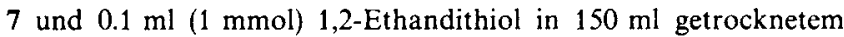
DMF wurden innerhalb von ca. $10 \mathrm{~h}$ bei $55^{\circ} \mathrm{C}$ in eine kräftig gerührte Suspension aus $650 \mathrm{mg}(2 \mathrm{mmol})$ Caesiumcarbonat in 11 DMF getropft. Nach Ende der Zugabe (DC-Kontrolle) wurde noch $1 \mathrm{~h}$ gerührt, das DMF i. Vak. bei $55^{\circ} \mathrm{C}$ entfernt und der gelbe Rückstand in $\mathrm{CHCl}_{3}$ oder Essigester aufgenommen. Die weitere Aufarbeitung erfolgt wie bei 10-12 [Methode a)]. Nach Einengen der organischen Phase wurde jedoch aus Ethanol oder Acetonitril umkristallisiert; Ausb. $120 \mathrm{mg} \mathrm{(26 \% ).}$

Allgemeine Vorschrift zur Darstellung $N, N^{\prime}$-geschützter 2,5-Dithia-13,14-diaza/6.2]paracyclophane (1K-VP in unpolaren Lösungsmitteln): In einer Einkomponenten-Verdünnungsprinzip-Apparatur nach Vögtle ${ }^{8 b)}$ wurde unter Stickstoff eine Lösung von $4 \mathrm{mmol}$ des Dibromids 7, 8 oder 9 und $4 \mathrm{mmol}$ des 1,2-Ethandithiols in $200 \mathrm{ml}$ trockenem Benzol innerhalb von $48 \mathrm{~h}$ zu einer heftig siedenden und kräftig gerührten Suspension aus $9.2 \mathrm{~g}$ Kaliumcarbonat, $300 \mathrm{mg}$ 18-Krone- 6 und 21 trockenem Benzol getropft. Nach Ende der Zugabe wurde noch eine weitere Stunde unter Rückfluß erhitzt. (DC-Kontrolle, gegebenenfalls wurde noch etwas Dithiol hinzugefügt.) Dann wurde vom Unlöslichen abfiltriert und zur Trockene eingeengt. Der gelbe Rückstand wurde in $\mathrm{CH}_{2} \mathrm{Cl}_{2}$ aufgenommen, einige Male mit 5proz. $\mathrm{NH}_{4} \mathrm{Cl}$-Lösung und schließlich mit Wasser gewaschen. Die Abtrennung der Polymeren erfolgte durch Kieselgelfiltration mit Petrolether/Essigester (4:1) als Laufmittel. Die weitere Reinigung wurde durch Mitteldrucksäulenchromatographie und anschließende Kristallisation aus Ethanol bzw. Essigester erreicht.

13,14-( Dimethylmalonyl)-2,5-dithia-13,14-diaza/6.2/paracyclophan (13): Ausb. $190 \mathrm{mg} \mathrm{(41 \% )} \mathrm{farblose} \mathrm{Nadeln,} \mathrm{Schmp.} 213^{\circ} \mathrm{C}$ $(\mathrm{EtOH}), R_{\mathrm{f}}=0.1$ (Petrolether/Essigester, 4:1). $-\mathrm{IR}(\mathrm{KBr}): v=$ $1690 / 1730 \mathrm{~cm}^{-1}(\mathrm{C}=\mathrm{O}), 1390(\mathrm{C}-\mathrm{N}), 720(\mathrm{C}-\mathrm{S}) .-{ }^{\mathrm{t}} \mathrm{H}-\mathrm{NMR}$ (300 MHz): $\delta=7.11$ (AA' $\mathrm{AB}^{\prime}$-System, $8 \mathrm{H}$, aromat. $\left.\mathrm{H}\right), 3.57(\mathrm{~s}, 4 \mathrm{H}$, benzyl. $\left.\mathrm{CH}_{2}\right), 1.97\left(\mathrm{~s}, 4 \mathrm{H}, \mathrm{CH}_{2}\right), 1.61\left(\mathrm{~s}, 6 \mathrm{H}, \mathrm{CH}_{3}\right) .-{ }^{13} \mathrm{C}-\mathrm{NMR}(300$ $\mathrm{MHz}): \delta=172.29(\mathrm{CO}), 140.07,132.95,129.54,128.59$ (aromat. C), 45.0 (tert. C), 37.46 (benzyl. $\left.\mathrm{CH}_{2}\right), 31.35\left(\mathrm{CH}_{2}\right) 21.9\left(\mathrm{CH}_{3}\right)$. - MS: $m / z(\%)=398(94)\left[\mathrm{M}^{+\cdot}\right], 306(20), 224(31), 175(23), 164(56)$, 146 (35), 132 (100), 90 (24).

$$
\mathrm{C}_{21} \mathrm{H}_{22} \mathrm{~N}_{2} \mathrm{O}_{2} \mathrm{~S}_{2} \text { Ber. } 398.1123 \text { Gef. } 398.1122 \text { (MS) }
$$

13,14-Succinyl-2,5-dithia-13,14-diaza/6.2]paracyclophan (14): Ausb. $154 \mathrm{mg}(40 \%)$ farblose Nadeln, Schmp. $275^{\circ} \mathrm{C}, R_{\mathrm{f}}=0.32$ (Toluol/Aceton, 4:1). $-{ }^{1} \mathrm{H}-\mathrm{NMR}(300 \mathrm{MHz}): \delta=7.08(\mathrm{~s}, 8 \mathrm{H}$, aromat. H), $3.54\left(\mathrm{~s}, 4 \mathrm{H}\right.$, benzyl. $\left.\mathrm{CH}_{2}\right), 3.0\left(\mathrm{~s}, 4 \mathrm{H}, \mathrm{CH}_{2} \mathrm{CO}\right), 1.94$ (s, $\left.4 \mathrm{H}, \mathrm{CH}_{2}\right) .-{ }^{13} \mathrm{C}-\mathrm{NMR}(300 \mathrm{MHz}): \delta=167.19$ (CO), 138.65, 
135.25, 129.16, 127.48 (aromat. C), 37.34 (benzyl. $\left.\mathrm{CH}_{2}\right), 31.35\left(\mathrm{CH}_{2}\right)$, $30.21\left(\mathrm{CH}_{2} \mathrm{CO}\right) .-\mathrm{MS}: m / z(\%)=384(82)\left[\mathrm{M}^{+\cdot}\right], 356(6), 292$ (10), 224 (9), 188 (100), 176 (8), 164 (30), 132 (46), 106 (60).

$$
\mathrm{C}_{20} \mathrm{H}_{20} \mathrm{~N}_{2} \mathrm{O}_{2} \mathrm{~S}_{2} \text { Ber. } 384.0966 \text { Gef. } 384.0966^{\circ} \text { (MS) }
$$

13,14-Glutaryl-2.5-dithia-13,14-diaza/6.2/paracyclophan (15): Ausb. $80 \mathrm{mg}(20 \%)$ farblose Nadeln, Schmp. $283^{\circ} \mathrm{C}$ (Zers.), $R_{\mathrm{f}}=$ 0.30 (Toluol/Aceton, $4: 1) .-{ }^{1} \mathrm{H}-\mathrm{NMR}(300 \mathrm{MHz}): \delta=7.34 / 7.04 /$ 6.84 (ABCD-System, 8H, aromat. H), 3.51 (AB-System, 4 H, benzyl. $\left.\mathrm{CH}_{2}\right), 3.17 / 2.70 / 2.45$ [AA'BB'CC'-System, $2 \mathrm{H}, \mathrm{CH}_{\mathrm{ax}} \mathrm{CO}(\mathrm{A}), 2 \mathrm{H}$, $\left.\mathrm{CH}_{\mathrm{eq}} \mathrm{CO}(\mathrm{B}), 2 \mathrm{H}, \mathrm{CH}_{2}(\mathrm{C}),{ }^{\prime} J_{\mathrm{AB}}=-12.8 \mathrm{~Hz}\right], 1.95 / 1.87\left(\mathrm{AA}^{\prime} \mathrm{BB}^{\prime}-\right.$ System, $\left.4 \mathrm{H}, \mathrm{CH}_{2}\right) .-{ }^{13} \mathrm{C}-\mathrm{NMR}(300 \mathrm{MHz}): \delta=171.39(\mathrm{CO})$, $137.89,136.22,129.32,129.02,127.74,123.94$ (aromat. C), 37.28 (benzyl. $\left.\mathrm{CH}_{2}\right), 32.64\left(\mathrm{CH}_{2} \mathrm{CO}\right), 31.15\left(\mathrm{CH}_{2}\right), 23.97\left(\mathrm{CH}_{2} \mathrm{CH}_{2} \mathrm{CH}_{2}\right)$. - MS: $m / z(\%)=398(100)\left[\mathrm{M}^{+\cdot}\right], 370(3), 306(10), 294(12), 209(16)$, $202(56), 174(32), 164(20), 132(41), 106(98)$.

$$
\mathrm{C}_{21} \mathrm{H}_{22} \mathrm{~N}_{2} \mathrm{O}_{2} \mathrm{~S}_{2} \text { Ber. } 398.11526 \text { Gef. } 398.11527 \text { (MS) }
$$

Abspaltung der Schutzgruppen zu 2,5-Dithia-13,14-diaza/6.2/paracyclophan-13-en (3). - Allgemeine Methode zur Abspaltung der Schutzgruppe: Eine Lösung von $1 \mathrm{mmol} 13$ bzw. 14 oder 15 in $100 \mathrm{ml}$ trockenem Dimethoxyethan wurde nach Zugabe von $680 \mathrm{mg}(10 \mathrm{mmol})$ Natriummethanolat $20 \mathrm{~h}$ unter Stickstoff bei Raumtemp. gerührt. Das Fortschreiten der Reaktion wurde dünnschichtchromatographisch überprüft. Nach Ende der Reaktion wurde der größte Teil des Dimethoxyethans i. Vak. verdampft, Eis/ Wasser zugegeben und mehrmals mit kleinen Portionen Essigester extrahiert (gegebenenfalls muß vor der Extraktion mit verdünnter $\mathrm{HCl}$ neutralisiert werden). Die Essigester-Phase wurde mit gesättigter $\mathrm{NaCl}$-Lösung gewaschen und mit $\mathrm{Na}_{2} \mathrm{SO}_{4}$ getrocknet. Nach Oxidation mit Luftsauerstoff oder mit $\mathrm{MnO}_{2}$ wurde 3 durch eine Kieselgelfiltration (zur Abtrennung polymerer Produkte) und anschließende Mitteldrucksäulenchromatographie mit Toluol als
Laufmittel gereinigt. Nach Einengen wurde 3 als gelber Feststoff erhalten; Ausb. $10-20 \%$, Schmp. $210^{\circ} \mathrm{C}, R_{\mathrm{f}}=0.2$ (Toluol). $-{ }^{1} \mathrm{H} \cdot$ NMR-, ${ }^{13} \mathrm{C}-\mathrm{NMR}$-, MS- und UV/VIS-spektroskopische Daten siehe Lit. $^{7}$.

\section{CAS-Registry-Nummern}

3: 121014-19-5 / 4: 114038-65-2 / 5: 22264-09-1 / 6: 121014-14-0/ 7: $114038-66-3$ / 8: 121014-15-1 / 9: 121014-16-2 / 13: 114038 67-4 / 14: 121014-17-3 / 15: 121014-18-4 / Dimethylmalonsäuredichlorid: 5659-93-8 / p-Hydrazotoluol: 637-47-8 / Bernsteinsäuredichlorid: 543-20-4 / Glutarsäuredichlorid: 2873-74-7 / 1,2-Ethandithiol: $540-63-6$

1) U. Dittrich, H.-F. Grützmacher, Chem. Ber. 118 (1985) 4404.

${ }^{2)}$ U. Dittrich, H.-F. Grützmacher, Chem. Ber. 118 (1985) 4414.

3) 3a) J. J. Gajewski, K. E. Gilbert, MMPMI, QCPE Indiana (1986). - ${ }^{36}$ R. M. Jarret, M. Saunders, STRPI, Yale Rapid Interactive Structure Input Program, Yale University 1983, modifiziert für MMPMI von J. J. Gajewski, K. E. Gilbert, Indiana 1986.

4) J. Schmiegel, H.-F. Grützmacher, Veröffentlichung in Vorberei-

5) 5a) B. Klieser, F. Vögtle, Angew. Chem. 94 (1982) 632. - ${ }^{\text {tb) F. }}$ F. Vögtle, F. Ley, Chem. Ber. 116 (1983) 3000.

${ }^{6)}$ U. Funke, Diplomarbeit, Univ. Bielefeld 1984.

7) U. Funke, H.-F. Grützmacher, Tetrahedron 45 (1987) 3787.

${ }^{8)}$ 8a) $^{\mathrm{K}}$. Ziegler in Methoden der Organischen Chemie (HoubenWeyl), Bd. IV $/ 22$, S. 733, Thieme, Stuttgart 1955. - ${ }^{8 b)} \mathrm{F}$. Vögtle, Chem.-Ztg. 96 (1972) 396.

${ }^{9)}$ B. Thulin, O. Wennerström, J. Somfai, B. Chmielarz, Acta. Chem. Scand., Ser. B, 31 (1977) 15.

${ }^{10)}$ H. Feuer, E. P. Rosenquist, F. Brown, jr, Isr. J. Chem. 6 (1968) 587.

$[35 / 89]$ 